\title{
Primary melanoma of the bladder: Case report and review of the literature
}

\author{
Francesco Barillaro ${ }^{1}$, Marco Camilli ${ }^{1}$, Paolo Dessanti ${ }^{2}$, Nader Gorji ${ }^{2}$, Fabio Chiesa ${ }^{3}$, \\ Alessandro Villa ${ }^{3}$, Alessandro Pastorino ${ }^{4}$, Carlo Aschele ${ }^{4}$, Enrico Conti ${ }^{1}$ \\ ${ }^{1}$ Department of Urology, ASL 5 Spezzino. Sarzana - La Spezia, Italy; \\ 2 Department of Pathology, ASL 5 Spezzino. La Spezia, Italy; \\ 3 Department of Radiology, ASL 5 Spezzino. Sarzana - La Spezia, Italy; \\ 4 Department of Oncology. ASL 5 Spezzino. La Spezia, Italy.
}

\begin{abstract}
Summary Skin melanoma represents one of the most common and lethal solid tumor. It usually develops on the skin but it can occur in any tissues with melanine-containing-cells (extracutaneous malignant melanoma). Only 4-5\% of malignant melanomas originate in extracutaneous tissues, and they have an extremely lethal behavior (1). These non-skin malignant melanomas are rare but extremely aggressive. Primary melanoma of the genitourinary tract accounts for less than $0.2 \%$ of all melanomas. To date only 28 cases of primary bladder melanoma (PMM) are described. We report a rare case of PMM of the bladder in a 72 years old man treated with radical cystectomy and immunotherapy with Nivolumab.
\end{abstract}

KEY WORDS: Bladder melanoma.

Submitted 16 April 2018; Accepted 29 April 2018 troscopy,colonscopy and a ophthalmologic exam ruled out the suspicious of a secondary lesion from a primitive malignant melanoma elsewhere. Patient's case was discussed by the institutional multidisciplinary uro-oncologic disease management team that stated a multimodal treatment. A radical cystectomy with a simple urinary diversion (UCS) and immunotherapy protocol were planned. Patient underwent surgery and the final histological exam reported a pT4 NO Mx R0 melanoma of the bladder (Figure 1). Immunoistochemical exam was positive for S-100, and SOX-10 and slightly positive for HMB45 while was negative for Cytocheratines CAM5.2, AE/1/AE3, 7, 20; Actine, CD34, Desmin,DOG-1, EMA, GATA-3, Myogenine, Chromoreanine, PSA and P63 (Figure 1).

Two months after surgery patient underwent contrast enhancement computed tomography (CeCT) without showing progression of the chest nodes. Three months

\section{Case report}

A 72 year-old caucasian man presented to the Emergency Room of our Hospital with gross asymptomatic haematuria. A cystoscopy showed a solid mass with red and brown spots in the surface, consequently the diagnosis of solid bladder tumor was posed. After obtaining informed consent, we performed a trans urethral resection of the bladder tumor (TURBt) without a radical debulking of the whole mass due to the aspect of extensive muscle invasion. The histological exam confirmed the diagnosis of PMM of the bladder. Patient denied an history of nevi-excision or other surgery.

A multiparametric Magnetic Resonance (mpMR) scan confirmed a solid tumor of $7 \times 6 \mathrm{~cm}$.

The lesion extended through all the bladder wall with suspicious iliac nodal metastasis. A fluorodeoxyglucose positron emission tomography computed tomography (FDG PET-CT) scan showed hypermetabolic capitation of the right ilar lymph-nodes and of the mesenteric tissue. A single capitation in para-vertebral region of the low portion of left lung was not clearly identified as metastasis. Dermatological exam, gas-
Figure 1.

Pathological specimens.

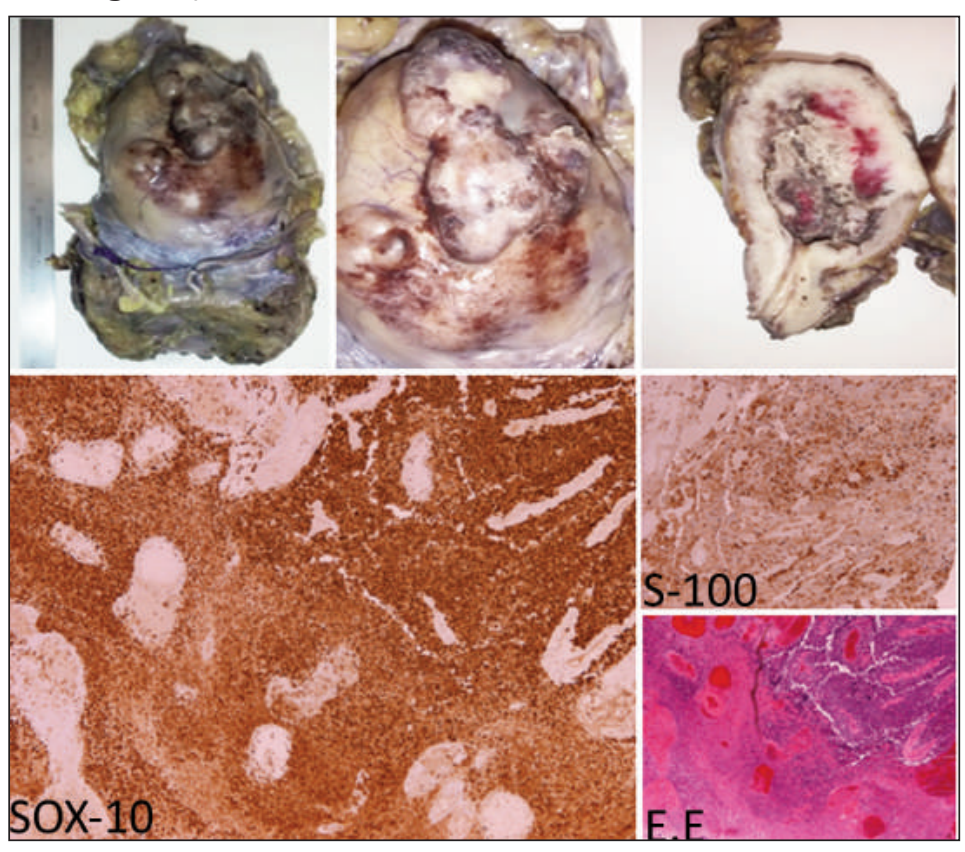

No conflict of interest declared. 
after surgery patient underwent immunotherapy with Nivolumab as for skin melanoma. A 14 day course of intravenous injections of $3 \mathrm{mg} / \mathrm{kg}$ of Nivolumab in 100 $\mathrm{ml}$ of saline solution was completed. A new FDG PET-CT scan six months after surgery showed a complete response of the nodal and mesenteric lesions while reported a decrease in size of the pulmonary lesion (17 $\mathrm{mm}$ vs $21 \mathrm{~mm}$ ). Thirteen months after surgery, a new FDG PET-CT showed a $4 \mathrm{~mm}$ small right inguinal nodal recurrence and a steady situation of the pulmonary lesion. Fifteen months after surgery patient is alive with Karnofsky score of 90 and ECOG 1-2.

\section{Discussion}

Primary melanoma of the urinary bladder is an extremely rare neoplasm and to date only 28 cases including this case are reported to medical literature (Table 1).

The diagnosis of primary melanoma of the bladder is not always easy. Ainsworth et al. (2) and Siroy and MacLennan (3) established some diagnostic criteria for primary bladder tumours:

(1) absence of any previous skin lesion, or (2) cutaneous malignant melanoma, or (3) primary visceral malignant melanoma, (4) recurrence pattern showing consistency with the primary tumour diagnosis, (5) atypical melanocytes at the tumour margin on microscopic examination.

Primary melanoma of the urinary bladder usually affects people over fifty and there is a slight prevalence in male sex $(60 \%)$. Our literature review presents few cases over a wide range of time with extremely different treatments and behavior. In addition, the follow up is extremely heterogeneous and only $12 / 28$ patients ( $42 \%$ ) were alive at time of the report with different follow-up (3-144 months; median 20.7 months). First choice treatment is surgery. The treatment can be conservative as trans urethral resection of the bladder tumor (TURBt) associated or not with endovesical Immunotherapy with Bacillus of Calmette Guerin (BCG); partial cystectomy can be considered as alternative conservative treatment. Radical Cystectomy can be carried out based on the staging of tumor and in patients with better performance status. Chemotherapy can be a possibility as unique therapy for patients who are poor surgical candidates and options include platinum-based chemotherapy as cisplatin/carboplatin plus paclitaxel.

Immunotherapy can be considered optional. Interferon and interleukin (IL)-2 are used for metastatic melanoma but the high tossicity and the low response rate reported in literature considerably limited the use.

Table 1.

Primary malignant melanomas reported in literature.

\begin{tabular}{|c|c|c|c|c|c|c|}
\hline & References & Age (y) & Sex & Treatment & Follow up (months) & Outcome \\
\hline 1 & Wheelock 1942 & 67 & $\mathrm{~F}$ & Partial cystectomy & 36 & Died \\
\hline 2 & Su and Prince 1962 & 61 & $F$ & None & 2 & Died \\
\hline 3 & Ainsworth et al. 1976 & 65 & $\mathrm{~F}$ & Radical cystectomy & 17 & Alive \\
\hline 4 & Willis et al. 1980 & 57 & $\mathrm{~F}$ & Radical cystectomy & 36 & Died \\
\hline 5 & Anichkov and Nikonov 1982 & 48 & M & Partial cystectomy & 12 & Died \\
\hline 6 & Anichkov and Nikonov 1982 & 46 & $\mathrm{M}$ & Radical cystectomy & 3 & Alive \\
\hline 7 & Ironside et al. 1985 & 56 & $M$ & None & 8 & Died \\
\hline 8 & Goldschmidt et al. 1988 & 53 & $\mathrm{~F}$ & Partial cystectomy & 7 & Died \\
\hline 9 & Goldschmidt et al. 1988 & 56 & $\mathrm{~F}$ & None & 6 & Alive \\
\hline 10 & Philippe et al. 1989 & 77 & $\mathrm{M}$ & TURB & n.r. & n.r. \\
\hline$\overline{11}$ & Van Ahlen et al. 1992 & 81 & $M$ & Radical cystectomy, radiotherapy, interferon-alpha & 24 & Died \\
\hline 12 & Lund et al. 1992 & 81 & $\mathrm{~F}$ & Local excision, radiotherapy chemotherapy & 15 & Alive \\
\hline$\overline{13}$ & Kojima et al. 1992 & 63 & $\mathrm{~F}$ & Chemotherapy & 18 & Died \\
\hline 14 & Lange-Welker et al. 1993 & 75 & M & Partial cystectomy & 3 & Died \\
\hline 15 & Mourad et al. 1993 & 34 & M & Radical cystectomy & 12 & Alive \\
\hline 16 & Niederberger and Lome 1993 & 53 & M & Radical cistectomy & 18 & Alive \\
\hline 17 & De Torres et al. 1995 & 44 & M & Radical cystectomy & 14 & Died \\
\hline 18 & Tainio et al. 1999 & 52 & M & TURB & 8 & Died \\
\hline 19 & Garcia Montes et al. 2000 & 44 & $\mathrm{~F}$ & TURB & 144 & Alive \\
\hline 20 & Khalbuss et al. 2001 & 82 & $\mathrm{~F}$ & Radiotherapy + cistectomy & 16 & Died \\
\hline 21 & T. Hsu and Y. Hsu 2002 & 73 & M & TURB + intravesical BCG and ReTURB at 2-7-9 months & 16 & Alive \\
\hline 22 & Baudet et al. 2005 & 7 & $\mathrm{~F}$ & Partial cystectomy & 84 & Alive \\
\hline 23 & Pacella et al. 2006 & 82 & M & TURB & 9 & Died \\
\hline 24 & Sundersingh et al. 2011 & 56 & M & Radical cystectomy and pelvic excision four months later & 10 & Alive \\
\hline 25 & El Ammari et al. 2011 & 71 & $\mathrm{M}$ & TURB & 5 & Died \\
\hline 26 & Truong et al. 2013 & 84 & $\mathrm{~F}$ & TURB + Ipilimumab & n.r & n.r. \\
\hline 27 & Otto et al. 2017 & 52 & M & TURB + Interferon/dacarbazine & 18 & Died \\
\hline 28 & Barillaro et al. 2018 & 72 & M & Radical cystectomy + Nivolumab & 16 & Alive \\
\hline
\end{tabular}


The fully human IgG4 PD-1 immune checkpoint inhibitor antibody Nivolumab has received the FDAapproval in March 2015 for squamous lung cancer treatment. In addition, antibodies targeting PD-1 or PD-L1 have demonstrated their efficacy and safety in additional tumors, including non-small cell lung carcinoma (NSCLC), renal cell carcinoma (RCC), bladder cancer, and Hodgkin's Lymphoma. Nivolumab plus Ipilimumab or Nivolumab alone, blocking the interaction between the programmed cell death PD1 and his ligand PD-Ll have been reported to be effective in antitumor response in melanoma $(4,5)$. Other treatment as radiation therapy might be only considered for palliative treatment in bladder melanoma. Based on our review TURBt or other conservative treatments were carried out in 14 patients (50\%) with a survival rate of $28.5 \%$ (4/14) in an average follow up of 14 months. One patient was treated with conservative treatment (TURBt) along with a combination of Interferon plus Dacarbazine and died after 18 months. In one case treated with TURBt and Ipilimumab survival was not reported.

Three cases did not receive any treatment and the reported follow up showed a death in 2 months and 8 months and a survival at 6 months. One case was treated with chemotherapy alone and died after 18 months. Radical cystectomy was carried out in 10 patients with median age of $59 \mathrm{y} / \mathrm{o}$; survival rate was $60 \%(6 / 10)$ at a median follow up of 15.5 months. Among patients treated with cystectomy, one received also radiotherapy and died 16 months after primary treatment. One patient received a multimodal treatment combined of cystectomy, radiotherapy and immunotherapy with interferon alpha and died 24 months after. In our case, Nivolumab was started as first line treatment after surgery based upon the BRAF, NRAS and c-KIT wild type molecular pattern. At time of this literature review is the only one reported with clinical and metabolic remission 15 months after primary treatment without relevant toxicities.

\section{ACKNOWLEDGMENTS}

All authors contributed equally to the manuscript drafting. The authors are the only ones responsible for the content and writing of the paper.

\section{Conclusions}

The best treatment of primary bladder melanoma is not univocally recognized due to the small number of cases reported in literature. While a bladder resection can be an option for localized small tumors, radical cystectomy may be the treatment of choice for invasive muscle disease.

As for metastatic skin melanoma, immunotherapy with Nivolumab seems representing a feasible therapy for this rare neoplasm.

The role of the surgical treatment versus only immune or chemotherapy is not known. According to literature review, radiation therapy seems not to be the treatment of choice. According to other cases reported, an almost total remission after 15 months after surgery and immunotherapy, can be considered a good therapeutic choice.

\section{REFERENCES}

1. Hussein MR. Extracutaneous malignant melanomas. Cancer Invest. 2008; 26:516-34.

2. Ainsworth AM, Clark WH, Mastrangelo M, Conger KB. Primary malignant melanoma of the urinary bladder. Cancer. 1976; 37:1928-36

3. Siroy AE1, MacLennan GT. Primary melanoma of the bladder. J Urol. 2011; 185:1096-7.

4. Mahoney KM, Freeman GJ, McDermott DF. The Next ImmuneCheckpoint Inhibitors: PD-1/PD-L1 Blockade in Melanoma. Clin Ther. 2015; 37:764-82.

5. Robert C, Long GV, Brady B, et al. Nivolumab in previously untreated melanoma without BRAF mutation. N Engl J Med. 2015; 372:320-30.

\author{
Correspondence \\ Francesco Barillaro, MD \\ francesco.barillaro@asl5.liguria.it \\ Marco Camilli, MD \\ marco.camilli@as15.liguria.it \\ Fabio Chiesa, MD \\ fabio.chiesa@asl5.liguria.it \\ Alessandro Villa, MD \\ dr.willaav@gmail.com \\ Enrico Conti, MD \\ enrico.conti@as15.liguria.it \\ Osp. S. Bartolomeo via Cisa, 19038 Santa Caterina, Sarzana (SP), Italy \\ Paolo Dessanti MD \\ paolo.dessanti@as15.liguria.it \\ Nader Gorji, MD \\ nader.gorji@as15.liguria.it \\ Alessandro Pastorino, MD \\ alessandro.pastorino@asl5.liguria.it \\ Carlo Aschele, MD \\ carlo.aschele@asl5.liguria.it \\ Osp. S. Andrea Via Vittorio Veneto 197, 19121 La Spezia (SP), Italy

